\title{
Regional and Local Temporal Trends of Borrelia burgdorferi and Anaplasma spp. Seroprevalence in Domestic Dogs: Contiguous United States 2013-2019
}

\author{
Jenna R. Gettings ${ }^{1 *}$, Stella C. W. Self ${ }^{2}$, Christopher S. McMahan ${ }^{3}$, D. Andrew Brown ${ }^{3}$, \\ Shila K. Nordone ${ }^{4}$ and Michael J. Yabsley ${ }^{1,5 *}$ \\ ${ }^{1}$ Southeastern Cooperative Wildlife Disease Study, Department of Population Health, College of Veterinary Medicine, \\ University of Georgia, Athens, GA, United States, ${ }^{2}$ Arnold School of Public of Health, University of South Carolina, Columbia, \\ SC, United States, ${ }^{3}$ School of Mathematical and Statistical Sciences, Clemson University, Clemson, SC, United States, \\ ${ }^{4}$ Department of Molecular Biomedical Sciences, Comparative Medicine Institute, College of Veterinary Medicine, North \\ Carolina State University, Raleigh, NC, United States, ${ }^{5}$ Warnell School of Forestry and Natural Resources, University of \\ Georgia, Athens, GA, United States
}

OPEN ACCESS

Edited by:

Luis E. Escobar,

Virginia Tech, United States

Reviewed by:

David V. Conesa,

University of Valencia, Spain

Antonio López-Quilez,

University of Valencia, Spain

${ }^{*}$ Correspondence: Jenna R. Gettings

jrgettings@gmail.com

Michael J. Yabsley

myabsley@uga.edu

Specialty section:

This article was submitted to

Parasitology,

a section of the journal

Frontiers in Veterinary Science

Received: 13 May 2020 Accepted: 17 September 2020

Published: 27 October 2020

Citation:

Gettings JR, Self SCW, McMahan CS,

Brown DA, Nordone SK and Yabsley MJ (2020) Regional and Local

Temporal Trends of Borrelia burgdorferi and Anaplasma spp.

Seroprevalence in Domestic Dogs: Contiguous United States 2013-2019.

Front. Vet. Sci. 7:561592.

doi: 10.3389/fvets.2020.561592
In 2019, in the United States, over 220,000 and 350,000 dogs tested positive for exposure to Anaplasma spp. and Borrelia burgdorferi, respectively. To evaluate regional and local temporal trends of pathogen exposure we used a Bayesian spatio-temporal binomial regression model, analyzing serologic test results for these pathogens from January 2013 to December 2019. Regional trends were not static over time, but rather increased within and beyond the borders of historically endemic regions. Increased seroprevalence was observed as far as North Carolina and North Dakota for both pathogens. Local trends were estimated to evaluate the heterogeneity of underlying changes. A large cluster of counties with increased B. burgdorferi seroprevalence centered around West Virginia, while a similar cluster of counties with increased Anaplasma spp. seroprevalence centered around Pennsylvania and extended well into Maine. In the Midwest, only a small number of counties experienced an increase in seroprevalence; instead, most counties had a decrease in seroprevalence for both pathogens. These trends will help guide veterinarians and pet owners in adopting the appropriate preventative care practices for their area. Additionally, B. burgdorferi and A. phagocytophilum cause disease in humans. Dogs are valuable sentinels for some vector-borne pathogens, and these trends may help public health providers better understand the risk of exposure for humans.

Keywords: Anaplasma spp., Borrelia burgdorferi, public health, vector-borne, veterinary epidemiology, ticks and tick-borne pathogens

\section{INTRODUCTION}

Dogs are frequently exposed to tick-borne pathogens, with Borrelia burgdorferi, agent for Lyme disease, and Anaplasma phagocytophilum, agent for granulocytic anaplasmosis, being among the most common $(1,2)$. The primary vectors for B. burgdorferi and A. phagocytophilum are Ixodes spp. Anaplasma platys is also implicated in canine anaplasmosis, but is presumed to be transmitted 
by Rhipicephalus sanguineus and causes cyclic thrombocytopenia $(3,4)$.

The incidence of human granulocytic anaplasmosis and Lyme disease has substantially increased $(5,6)$ in recent decades, due in part to increased recognition and reporting practices (7). Increasing incidence and geographical distribution $(6,8,9)$ are also believed to be related to changes in the distribution and densities of the tick vector Ixodes spp., changes in the reservoir host communities, and the interaction between humans and the vector (10-12). The current emergence of B. burgdorferi is believed to have started in the 1970s. At that time, previously farmed regions were abandoned as the populace migrated back into cities, and these areas became reforested (10). Reforestation has led to a rise in deer populations, an important host for adult Ixodes spp. Further, habitat fragmentation (e.g., suburbanization) has led to an increase in edge habitat which is ideal for small mammals, such as mice, which play a critical role in the enzootic transmission of $A$. phagocytophilum and $B$. burgdorferi. Moreover, the biodiversity of these animal host communities influence the prevalence of infection in ticks (13). Collectively, these changes have lead to an increase in tick populations, and their presence has expanded. For example, counties reporting the presence of Ixodes spp. ticks rose from 1,058 in 1998 to 1,531 in 2016 within the contiguous United States (11).

Given the close association between human and canine vector-borne diseases (14-16), the rising number of cases observed in humans and the documented expansion of the vector range raise the question as to whether canine vectorborne disease is increasing as well. Recently, a Bayesian spatio-temporal binomial regression model was developed to describe the temporal trends in these seroprevalence data (17). In that study, the canine seroprevalence of B. burgdorferi was found to be increasing. In this study, we explore this further for B. burgdorferi using an expanded and more contemporary dataset and apply the model to canine Anaplasma spp. seroprevalence in order to identify increases in canine Anaplasma spp. or B. burgdorferi seroprevalence within the United States.

Recognizing areas of increasing canine seroprevalence is of importance when considering recommendations for preventative care. There are several interventions recommended by veterinarians to reduce the risk of exposure and infection. These include use of acaricides, vaccination against B. burgdorferi, regular examination for the presence of ticks, and avoidance of known tick habitats (18). Uncertainty of the level of risk confounds recommendations and compliance with testing and preventative interventions. Clinicians acting on historical experience may fail to initially recognize the increased risk. As such, understanding the dynamic nature of vectorborne disease and change in prevalence is imperative for prevention and control. Furthermore, the pet dogs represented in these data share a peridomestic environment with their human counterparts. The information from these trends can be applied to the One Health approach to the control and prevention of vector-borne disease in humans and animals alike.

\section{METHODS}

\subsection{Data}

Over 30 million test results for Anaplasma spp. and B. burgdorferi were collected from point-of-care SNAP $\AA 4 D x \circledast$ Plus (IDEXX Laboratories, Inc. Westbrook, ME) tests, a qualitative modified enzyme-linked immunosorbant assay (ELISA), performed inclinic and at regional reference laboratories between January 2013 and December 2019 (1). Figures 1A,B depict an aggregation of the Anaplasma spp. and B. burgdorferi data, respectively, from January 2013 to December 2019. Displayed are the overall raw seroprevalences for each county (the number of seropositive tests divided by the total number of tests reported) over the study period, with white counties being those which did not report any test results. This data presents with strong spatial dependence ( $p$-value for Moran's I statistic $<0.0001$ for both datasets).

The test detects antibodies against Anaplasma spp. and $B$. burgorferi as well as Ehrlichia spp. (agents of ehrlichiosis) and antigen from Dirofilaria immitis (causative agent of canine heartworm disease). It is used commonly by veterinarians throughout the United States for annual screening during wellness examinations or for diagnosis of suspected vectorborne illness. Results were collated automatically by IDEXX Laboratories into a centralized database, from which aggregate data were provided to the investigators at a county and monthly scale. Importantly, the reported county is that of the clinic and no patient histories are known. Anaplasma spp. exposure is determined by the presence of antibodies to the major surface protein-2 (p44) of Anaplasma phagocytophilum (19). Anaplasma platys has been shown to cross-react to p44 $(20,21)$, and seroconversion for both occurs 10-14 days post-infection (21). For B. burgdorferi, the ELISA detects antibodies that are seroreactive to the C6 peptide which is based on invariable region 6 , a highly immunogenic and conserved region on the outer membrane protein VlsE of B. burgdorferi (22). Seroconversion to C6 occurs after the infection has become disseminated and can occur as early as 3-4 weeks (23).

\subsection{Model Definition}

Regional and local trends were estimated using a Bayesian spatio-temporal binomial regression model. Let $y_{s t}$ and $n_{s t}$ be the number of seropositive tests and total number of tests, respectively, from county $s$ in month $t$. We assume

$$
y_{s t} \mid n_{s t}, p_{s t} \sim \operatorname{Binomial}\left(n_{s t}, p_{s t}\right)
$$

where $p_{s t}$ is the unknown seroprevalence in county $s$ during month $t$. Thus, we are assuming that given the number of tests and seroprevalence, the number of seropositive tests in county $s$ and month $t$ follows a binomial distribution with the number of trials equal to the number of tests and the probability of success on each trial equal to the seroprevalence. We model the seroprevalence $p_{s t}$ using a linear predictor $\eta_{s t}$ :

$$
g^{-1}\left(p_{s t}\right)=\eta_{s t}=v+\beta(s) t+\xi_{s t}
$$

Here $g(\cdot)$ is the logistic function and ensures $p_{s t}$ will be between 0 and $1, v$ is a global intercept term, $\beta(s)$ is the regional trend 

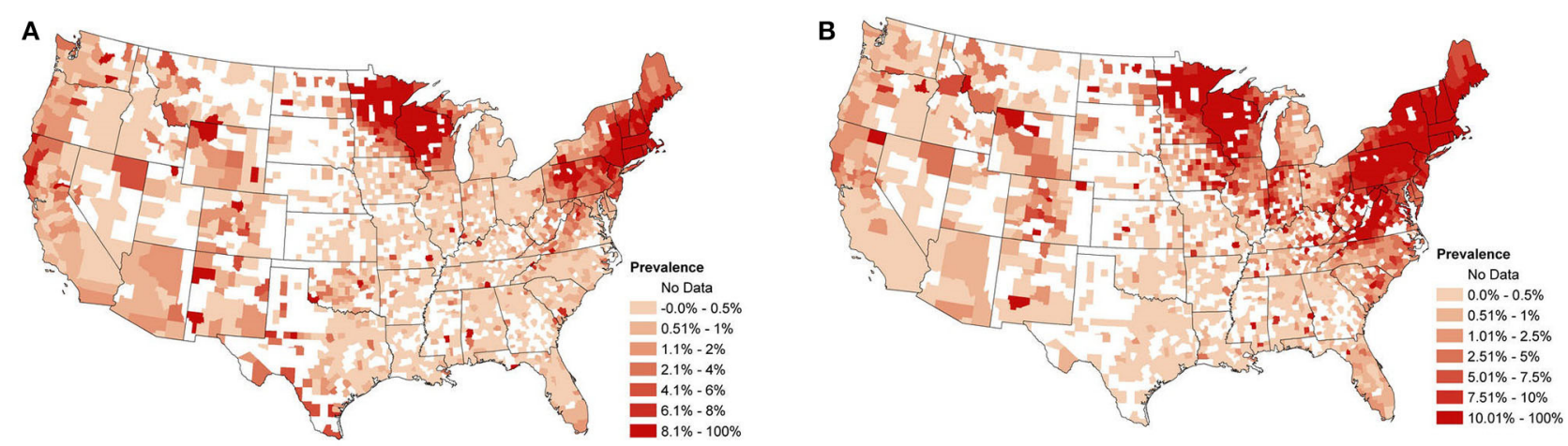

FIGURE 1 | (A) Overall observed canine Anaplasma spp. seroprevalence from January 2013 to December 2019. Calculated as the proportion of positive test counts among all test counts within a county for the study period. (B) Overall observed canine B. burgdorferi seroprevalence from January 2013 to December 2019. Calculated as the proportion of positive test counts among all test counts within a county for the study period.

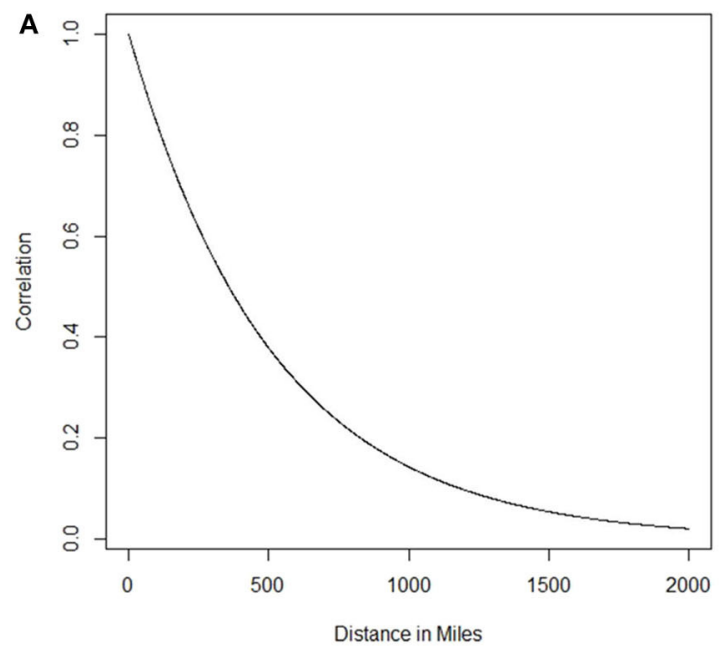

Anaplasma spp.

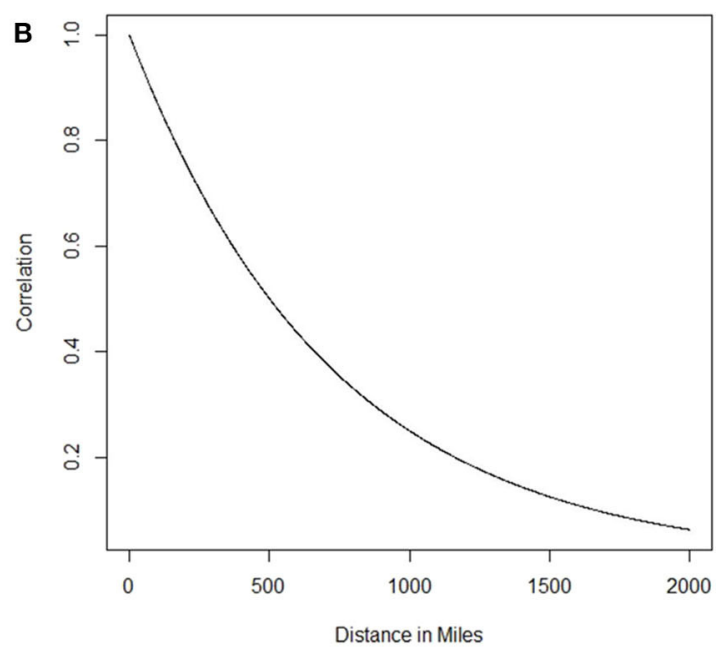

Borrelia burgdorferi

FIGURE 2 | The correlation between the trend parameter for a given county and any other county as a function of distance for Anaplasma spp. seroprevalence and Borrelia burgdorferi seroprevalence is depicted in $(\mathbf{A}, \mathbf{B})$, respectively. The curve represents the decaying influence that any other county has on a given county as the distance between them increases.

parameter at county $s$, and $\xi_{\text {st }}$ is a spatio-temporal random effect included to account for the spatio-temporal dependence in the data. Here $t$ denotes time in months, rescaled to be between 0 and 1 for numerical reasons. The $\beta(s)$ 's follow a Gaussian predictive process, which ensures the regional trends change smoothly over space and allows the model to use information from a relatively large surrounding area to estimate the trend at each county. The parent process for the GPP was defined on 100 knot locations, with a mean function $\boldsymbol{\mu}\left(\boldsymbol{\ell}_{s}\right)=$ 0 , and covariance function $\sigma^{2} \rho\left(\boldsymbol{\ell}_{s}, \boldsymbol{\ell}_{s^{\prime}} ; \boldsymbol{\theta}\right)=\sigma^{2} \theta^{d_{s, s^{\prime}}^{2}}$, where $\boldsymbol{\ell}_{s}$ and $\boldsymbol{\ell}_{\boldsymbol{s}^{\prime}}$ denote the latitude-longitude of the locations of knot $s$ and $s^{\prime}$, respectively, $\theta \in(0,1), \sigma^{2}>0$ and $d_{s, s^{\prime}}$ denotes the euclidean distance between locations $s$ and $s^{\prime}$. Such a covariance function allows the strength of the correlation between two observations to decrease as the distance between them increases (see Figure 2). For additional details, including information regarding the selection of the knot locations, see the Appendix and (17). For more on Gaussian predictive processes, see (24). The data display heavy spatio-temporal dependence. Neglecting to model this dependence can lead to inaccurate estimation and inference. The $\xi_{s t}$ 's are designed to account for this spatio-temporal dependence through a vector autoregession. Specifically, letting $\boldsymbol{\xi}_{t}=\left(\xi_{1 t}, \xi_{2 t}, \ldots, \xi_{S t}\right)^{\prime}$ be the vector of spatio-temporal random effects from all counties at time $t$, we assume

$$
\begin{aligned}
& \boldsymbol{\xi}_{1} \sim N\left(\mathbf{0}, \tau^{2}(\boldsymbol{D}-\omega \boldsymbol{W})^{-1}\right) \\
& \boldsymbol{\xi}_{t} \sim N\left(\zeta \boldsymbol{\xi}_{t-1}, \tau^{2}(\boldsymbol{D}-\omega \boldsymbol{W})^{-1}\right) \text { for } t \geq 2,
\end{aligned}
$$


where $\zeta \in(-1,1)$ is a temporal correlation parameter, $\tau^{2}>0$ is a variance parameter, $\omega \in(0,1)$ is a propriety parameter included to ensure the invertiblity of the covariance matrix, $W$ is the $S \times S$ adjacency matrix for the counties (i.e., $W_{s s^{\prime}}=1$ if counties $s$ and $s^{\prime}$ share a border and 0 otherwise), and $\boldsymbol{D}$ is a diagonal matrix with $\boldsymbol{D}_{s s}=\sum_{j=1}^{S} \boldsymbol{W}_{s j}$. The autoregressive structure of our model is similar to that found in (25) and (26), which was based on a generalization of the common conditional autoregressive (CAR) model; for more on CAR models, see (27), (28), or (29). Markov chain Monte Carlo (MCMC) methods were used to fit the model by sampling the unknown parameters from their posterior distributions; with posterior estimation and inference proceeding in the usual manner. Let $\eta_{s t}^{(g)}$ denote the value of the linear predictor calculated using the parameter values from the gth MCMC sample. To estimate the local trends for county $s$, we fit the following ordinary least squares model for each value of $g$ :

$$
\eta_{s t}^{(g)}=\alpha_{0 s}^{(g)}+\alpha_{1 s}^{(g)} t+\epsilon_{s t}^{(g)} \text { for } t=1, \ldots, T
$$

where the $\epsilon_{s t}^{(g) \text { 's }}$ are independent and identically distributed normal random errors. For each county $s$,

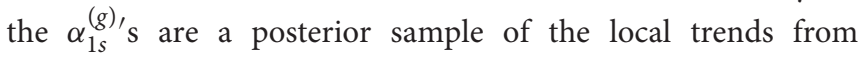
county $s$.

In conducting this analysis, a few additional details are of note. First, the data under study presents with county-month pairs for which no tests are reported; e.g., see Figures 1A,B. The spatio-temporal structure in the selected model makes it robust to this sort of missing data, as was demonstrated in (17). Second, to implement the proposed model, a custom MCMC sampling routine was developed and coded in $\mathrm{R}$. This code is available on GitHub (see link at the end of manuscript). Model fitting took approximately $12 \mathrm{~h}$ for the Anaplasma spp. data and 18 $\mathrm{h}$ for the B. burgdorferi data on a desktop computer running Windows 10 with an Intel(R) Xeon(R) E-2186G CPU with a 3.80 Ghz processor. For more information on the model, including an explanation of the difference between local and regional trends, details concerning how the model handles missing data, the prior distributions used, and the MCMC sampling procedure, see the Appendix and (17).

\subsection{Model Selection Procedure}

To identify the final model for both datasets, we fit several variants of the model above; e.g., with and without spatiotemporal random effects, with and without spatially varying trends, etc. The deviance information criteria (DIC) was used to select the "best" model from among these candidates. The full model described above had the lowest DIC, and was selected as our final model. To assess the predictive efficacy of these models, the area under the curve (AUC) of a receiver operating characteristic (ROC) curve $(30,31)$ was computed. That is, the ROC curves were produced for both the B. burgdorferi and Anaplasma spp. models by plotting the sensitivity (proportion of positive tests correctly predicted) by one minus the specificity (proportion of negative tests correctly predicted) and the area under these curves were computed to determine AUC. In general, an AUC value of 0.70 or greater is considered to be an acceptable model (32).

\section{RESULTS}

\subsection{Model Accuracy}

The AUC for the B. burgdorferi trends model was 0.80 . The AUC for the Anaplasma spp. model was 0.85 . Both were $>0.70$, indicating good predictive performance.

\subsection{Regional Temporal Trends}

The analysis presented in this manuscript provides two perspectives of the changing seroprevalence of B. burgdorferi and Anaplasma spp. in dogs within the contiguous United States. The regional trend for each county is based on data from a relatively large surrounding area, with the influence of the data from nearby counties diminishing with distance. For a formal depiction of how influence decreases with distance, see Figure 2. Three groups were chosen and designated as high (correlation above 0.75), moderate (correlation between 0.75 and 0.5 ), and low (correlation $<0.5$ ) influence. The distances that correspond to these correlation values based on Figure 2, respectively, are 0-147 miles, 148-357 miles, and >357 miles for Anaplasma spp. and 0-207 miles, 208-501 miles, and >501 miles for B. burgdorferi. In reality, the influence diminishes in a continuous fashion as distance increases as depicted in Figure 2.

\subsubsection{Anaplasma spp.}

The regional change in canine Anaplasma spp. seroprevalence is shown in Figure 3A. Displayed are the posterior mean values of the temporal trend parameter $\beta(s)$ from equation (2) for each county. Positive values represent an increase in seroprevalence between January 2013 and December 2019, while negative values represent a decrease. Figure 3B shows only the counties deemed to have a relevant positive increase in regional seroprevalence. Herein, the importance of model parameters are determined based on whether their corresponding credible interval captures 0 or not; for further discussion on the interpretation of credible intervals [see (33)]. As is always the case, when simultaneously assessing the relevance of multiple parameters one should be aware of the multiplicity issue (34, 35). The highest positive regional trends are centered in two areas: New England and northern Wisconsin and Minnesota. The surrounding regions experienced smaller changes. In the eastern US, increasing seroprevalence is seen as far as southern Virginia and extends toward the Ohio border. In the Midwest, increasing seroprevalence extends into North Dakota and Iowa. Two foci with smaller positive trends are present in northern California and southern Oregon and in southern and western Texas.

\subsubsection{B. burgdorferi}

From Figure 4, we see that the areas with positive regional trends for B. burgdorferi seroprevalence were similar to those for Anaplasma spp., but with a larger distribution. In the eastern US these trends extend into North Carolina, Tennessee, Kentucky, and Ohio. In the Midwest, these areas extend into Iowa, Illinois, 

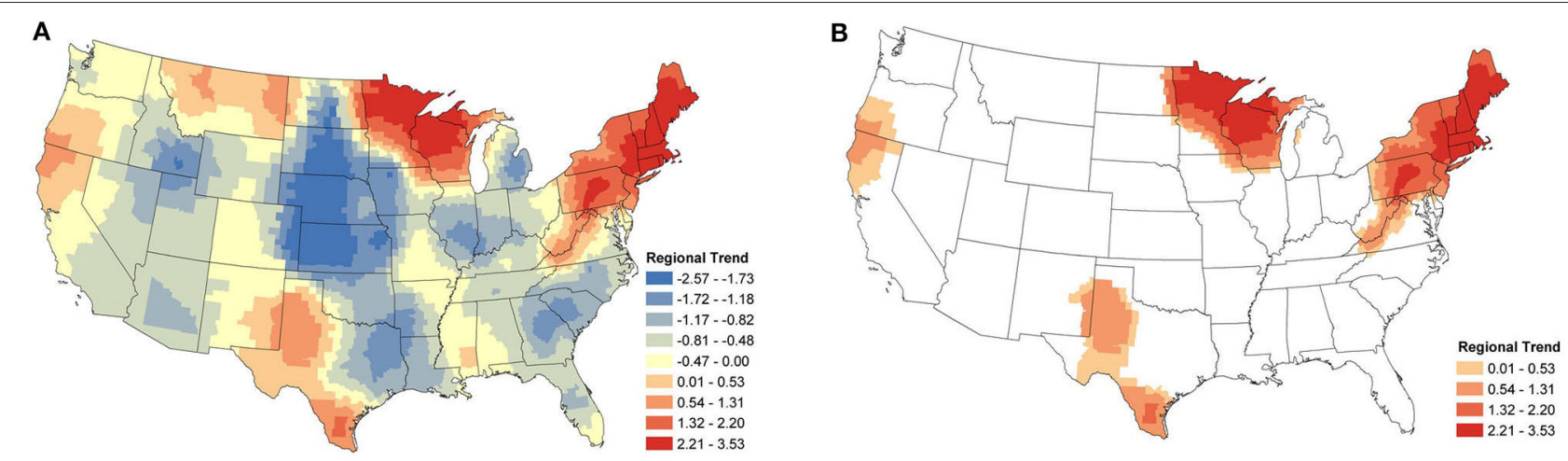

FIGURE 3 | Anaplasma spp. Regional Seroprevalence Temporal Trends: (A) posterior means of the regional temporal trend parameter $\beta(s)$ from Equation (2); (B) counties with a relevant positive trend parameter $\beta(s)$ based on $95 \%$ credible intervals. Supplementary Figure 2 depicts the lower and upper bounds of $95 \%$ credible intervals for the regional trends from each county.
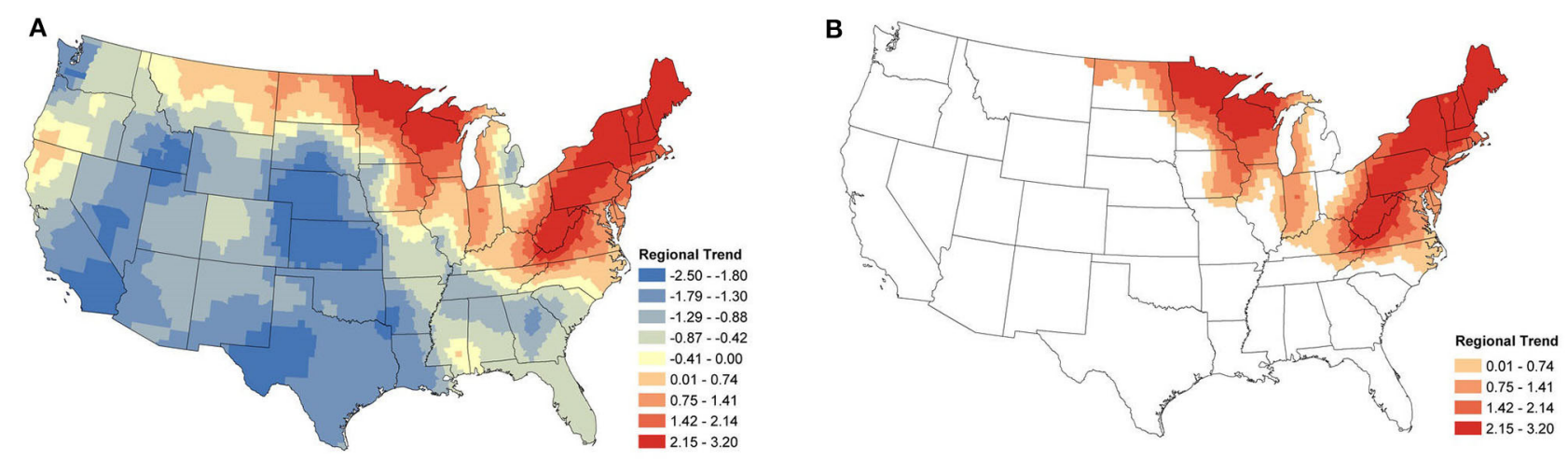

FIGURE 4 | B. burgdorferi Regional Seroprevalence Temporal Trends: (A) posterior means of the regional temporal trend parameter $\beta(s)$ from Equation (2); (B) counties with a relevant trend parameter $\beta(s)$ based on $95 \%$ credible intervals. Supplementary Figure 2 depicts the lower and upper bounds of $95 \%$ credible intervals for the regional trends from each county.

Indiana, Michigan, and North Dakota. No relevant increase in seroprevalence is observed along the West coast (Figure 4B).

The focus of the regional study was positive trends, identifying areas where seroprevalence has increased. However, negative trends can also be identified and for completeness, these maps are available in the Supplementary Material (Supplementary Figure 1). Briefly, areas outside of endemic regions experienced little to no changes. Areas that appear to have large negative trends (e.g., central USA) are areas with very low prevalence (nearly $0 \%$ ), and consequently, even small changes can create a relatively large trend.

\subsection{Local Temporal Trends}

Local temporal trends were also obtained from the same model and reveal the underlying spatial heterogeneity in the changes in seroprevalence (Figures 5A, 6A). These trends are driven predominately by the data from within a single county. As a result, trends can be markedly different, even between neighboring counties. Patterns in the clusters of relevant positive and negative trends are of greatest interest in understanding the local changes.

\subsubsection{Anaplasma spp.}

Focusing on the counties with relevant positive estimates for Anaplasma spp. seroprevalence, a large cluster of strongly positive trends were present throughout the Northeast (Figure 5B), with a large area centered within Pennsylvania. Positive counties are seen as far south as Virginia and Kentucky. Perhaps of most interest is the lack of a large cluster of positive trends in Minnesota and Wisconsin, where only a handful of counties are positive. Most of the counties with negative estimates are scattered with little clustering, suggesting local drivers of the trend, but the clusters within North Carolina, Michigan, and much of the Midwest without the intermingling of positive counties does suggest that these areas may have experienced some decrease in seroprevalence in the last several years.

\subsubsection{B. burgdorferi}

Figure 6B shows the relevant local temporal trends for $B$. burgdorferi seroprevalence. The largest cluster of positive trends was centered around the West Virginia and Ohio border, suggesting that this area saw the most change during the study period. This cluster extended into Maine and outward into North 

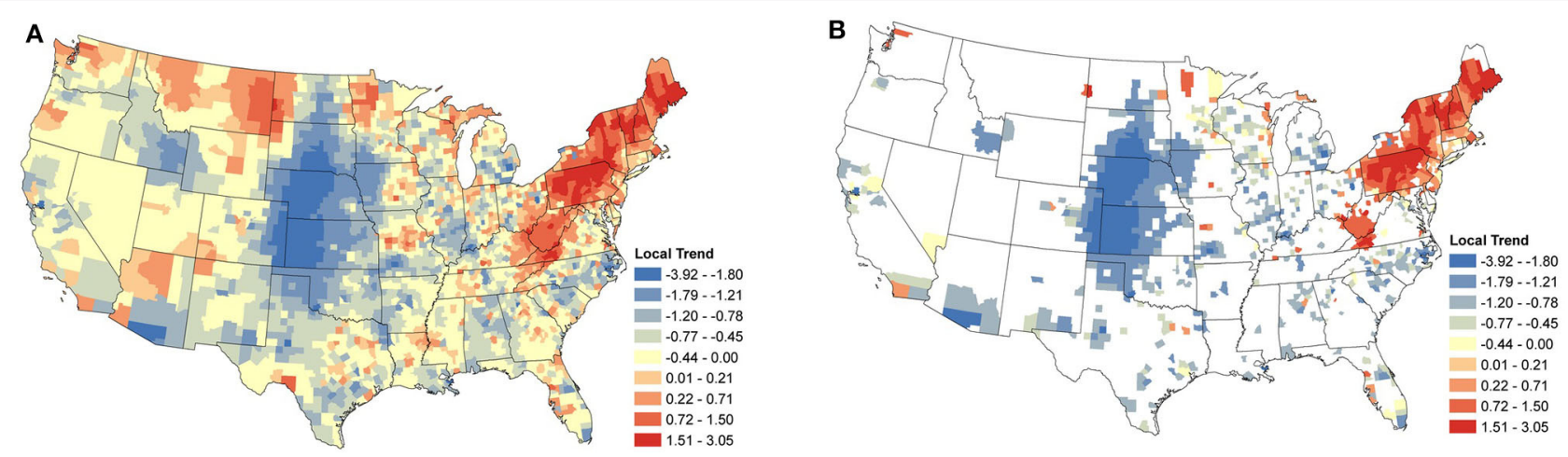

FIGURE 5 | Anaplasma spp. Local Seroprevalence Temporal Trends: (A) posterior means of the local temporal trend parameter, $\alpha_{1 s}^{(g)} \mathrm{s}$, from Equation (3) for all counties; (B) posterior means of the positive local temporal trend parameter for counties in which the $95 \%$ credible interval did not contain zero.

Supplementary Figure 3 depicts the lower and upper bounds of 95\% credible intervals for the regional trends from each county.
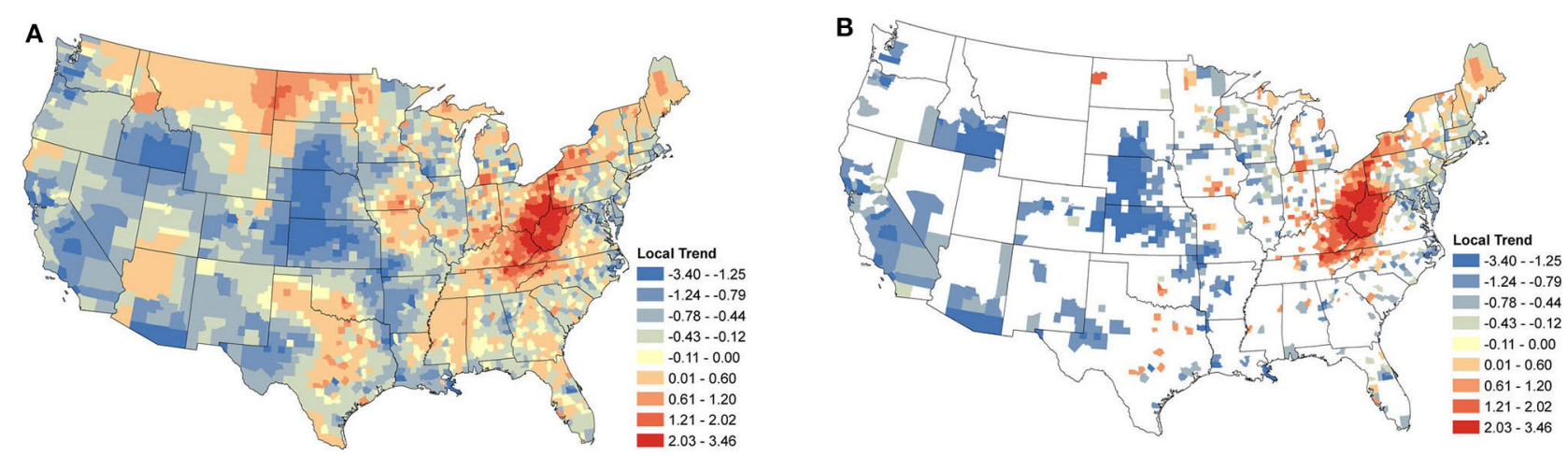

FIGURE 6 | Borrelia burgdorferi Local Seroprevalence Temporal Trends: (A) posterior means of the local temporal trend parameter, $\alpha_{1 \mathrm{~s}}^{(g)}$ s, from Equation (3) for all counties; (B) posterior means of the positive local temporal trend parameter for counties in which the $95 \%$ credible interval did not contain zero.

Supplementary Figure 3 depicts the lower and upper bounds of 95\% credible intervals for the regional trends from each county.

Carolina, Kentucky, Tennessee, and Southwestern Ohio. Looking to the Midwest, a loose cluster of positive trends can be seen throughout the Midwest with the larger clusters in Iowa and southwestern Michigan. Negative trends were evident in the majority of the counties along the New England coast extending toward the west. The negative trends also mirrored those of Anaplasma spp. in the Midwestern states, particularly Wisconsin and Illinois. Large clusters of negative trends were also scattered throughout the rest of the USA, including the west coast. This was not surprising for the same reason that the regional trends were also negative in many of these areas.

\section{DISCUSSION}

The temporal trends of canine Anaplasma spp. and $B$. burgdorferi seroprevalence are described at two different levels of aggregation: regional (multi-state) and local (county). The broader regional trends provide us with an overview of how the prevalence of these pathogens has changed for a general population of dogs over a large spatial area, while the localized county-level trends provide an estimate for the level of risk for populations of dogs within a county. Neither can ascribe a specific risk to an individual dog, but both provide information on how risk is changing over time. It is important to note that these results are relative to the underlying seroprevalence. As such, practitioners are advised to examine seroprevalence at their state and regional level to assess underlying seroprevalence of pathogens and evaluate patient risk (1). For example, there may be regions that do not show an increasing trend; however, the baseline prevalence of a pathogen could be relatively high (but stable).

\subsection{Regional Temporal Trends}

The regional trends analysis show that canine seroprevalence for both Anaplasma spp. and B. burgdorferi increased broadly in the Northeastern and upper Midwestern states from 2013 to 2019. These changes are likely influenced by the same factors that are driving changes in the geographical distribution and density of I. scapularis. The distribution and density of this tick are known to be expanding across a variety of geographical ranges 
in the United States and Canada $(36,37)$. This is believed to be predominately driven by climate change that supports vector viability, increases in white-tailed deer population densities, and habitat change such as reforestation and fragmentation (38). In contrast, within the range of $I$. pacificus, little change was seen in Anaplasma spp. and B. burgdorferi seroprevalence. The only exception was northern California and southern Oregon, where Anaplasma spp. seroprevalence increased with relevance. This relative lack of change is supported by the stable population of I. pacificus, suggesting that this region is not experiencing the same change as that in the Northeast and Midwest (11).

In most areas, the trends in canine Anaplasma spp. and $B$. burgdorferi seroprevalence were similar, with B. burgdorferi having a greater geographical distribution. This is consistent with molecular surveys of ticks, molecular testing of Peromyscus leucopus (white-footed mouse) reservoirs, and serologic data from dogs. Several molecular studies on I. scapularis have shown that the prevalence of $B$. burgdorferi is generally higher than for A. phagocytophilum (39-41). Similarly, studies in P. leucopus have shown higher prevalences for $B$. burgdorferi compared to A. phagocytophilum; for example, prevalence in Wisconsin and Minnesota was 24 vs. $1.7 \%$ and 42 vs. $20 \%$, respectively $(42,43)$. Finally, the seroprevalence of $B$. burgdorferi is much higher in dogs compared with Anaplasma spp. $(1,44)$. In areas extending out from the historically highly endemic regions, the prevalence of Anaplasma spp. in Ixodes spp. may be below the level of detection and thus impact resolution of change described by this model (45). This, in turn, may explain the disproportionately larger geographical distribution of increased B. burgdorferi seroprevalence. However, while lower prevalence of these pathogens in Ixodes spp. and important reservoirs helps explain some of the differences we observe in these trends, it would be an over-simplification to not consider the myriad of other factors that impact the ecologies of Anaplasma spp. and $B$. burgdorferi (46). In addition, the sensitivity of the currently used Anaplasma (SNAP $\AA 4 \mathrm{Dx} \circledast \mathrm{B}$ lus) antigen (APH1 ) is lower compared with the B. burgorferi C6 peptide which may also explain some differences in prevalence due to missed cases (47-49).

One limitation of our Anaplasma spp. data is that the diagnostic test (SNAP $₫ 4 \mathrm{Dx}(\mathrm{P}$ Plus) used to measure antibody against Anaplasma spp. does not differentiate between $A$. phagocytophilum and A. platys. These two pathogens have different expected geographic distributions based on their vectors; however, there are areas that may overlap, particularly in the western United States. Rhiphicephalus sanguineus (brown dog tick) (50), the presumed vector of $A$. platys, has a broad geographical distribution that is facilitated in part by its endophilic (indoor dwelling) nature and affinity for domestic dog hosts (51). We are limited in our knowledge of the precise distribution and density of $R$. sanguineus and so the degree to which it overlaps with the ranges of I. scapularis and I. pacificus is not fully understood. Unlike the potentially broad distribution of A. platys, the primary Anaplasma sp. expected in dogs is A. phagocytophilum, which is restricted to the range of its vectors Ixodes scapularis and I. pacificus. Thus, A. phagocytophilum is believed to account for the trends observed in the northeastern and upper Midwestern regions, and some trends along the western coast.

In this study, areas of increased seroprevalence that may have been impacted by $A$. platys include small regions in California and Oregon and southern Texas. In California and Oregon, increased seroprevalence was observed for Anaplasma spp., but not B. burgdorferi. This may be due in part to the presence of A. platys, but we should also consider the variable diversities of Ixodes spp. (52) and their host communities (53) in this region compared to the Northeast and Midwest that may also impact the risk of exposure to dogs. In Texas, Qurollo et al. reported that dogs were equally likely to be exposed to A. platys and A. phagocytophilum (seroprevalences of 2.0 vs. $2.2 \%$, respectively) (2). This is in contrast to the Northeast where dogs are more frequently exposed to A. phagocytophilum (1.5 vs. 13.0\%). Given the differences between the vectors of the two Anaplasma spp. and the pathogens those vectors carry, future studies would benefit greatly from distinguishing the temporal trends of the two Anaplasma spp.

\subsection{Local Temporal Trends}

When the analysis of data shifts from regional trends to local trends, we observe useful information for veterinary practitioners concerned with changes at the county level. At this finer spatial resolution, we are able to see how seroprevalence trends can change over small distances, highlighting the importance of interpreting aggregated data with care. The local trends also appear to highlight areas in which exposure may be newly emergent. Note the trends for B. burgdorferi seroprevalence in West Virginia, Ohio, and Kentucky (Figure 6B).

In the Northeast and Middle Atlantic states, we observed a large cluster of counties with increasing local seroprevalence for both Anaplasma spp. and B. burgdorferi. However, the centers of these clusters were in different locations. The positive trends for B. burgdorferi were centered around West Virginia (Figure 6B). This cluster extended in most directions and positive counties were scattered throughout the Northeast, but notably not along the coast. Similar results for canine seroprevalence were obtained by (54) but the main clusters of counties with increasing trends were slightly further north which is likely because their canine serologic data set ended in 2017, 2 years prior to our final year of sampling (54). A recent report of temporal changes in human Lyme disease prevalence mirrors our observations to some extent, although the goals of the two studies differed. Using the first year of detection of human cases and select environmental and demographic factors in their analysis, Bisanzio et al. (55) identified West Virginia as a state with numerous new county reports from 2000 to 2017 and concluded the spread velocity of human Lyme disease estimated by their model was faster in the South. The approach Bisanzio et al. was to analyze the spread of human infections and the likelihood a county would become positive in a given year, but our approach differs in that our model provides data on trends in prevalence for both endemic and non-endemic regions (55). This explains why we had regions of increasing prevalence (e.g., in the Northeast and parts of upper Midwest) that do not show up on maps in Bisanzio et. al as being high risk (because they were already endemic). Thus, differences 
in the two analyses are likely attributable to four main factors; (1) modeling difference (two step diffusion model vs. a spatiotemporal binomial regression model with spatially varying trend parameters), (2) population under study (dog vs. human), (3) goals of study (predicting first case vs. identifying trends), and (4) the time range over which the study was conducted.

In our analysis, the cluster of counties with increasing Anaplasma spp. seroprevalence was centered around Pennsylvania and extended northward through Maine. Similar increasing trends from this same region were reported between 2010 and 2017 by (54). Differences in the prevalence of infection of B. burgdorferi and Anaplasma spp. in Ixodes spp. in these two regions might explain some of this difference, but the prevalence of infection of I. scapularis in West Virginia is not known at this time. There is evidence of the expanding range of Ixodes spp., with $B$. burgdorferi $(8,9,45,56)$, but as discussed with respect to regional trends, Anaplasma spp. may be lagging due to limitations in detection of accurate pathogen prevalence or other factors (45).

Local trends have direct application to veterinary medical decisions. The spatial difference in the temporal trends of B. burgdorferi and Anaplasma spp. pathogens may be related to differences in preventative practices. Duration of endemicity, awareness of acaricide products, socioeconomic factors, and client-based education vary substantially between endemic and non-endemic regions of the country, thereby affecting the frequency of preventative use (57). In addition, there are currently no protective vaccines on the market for Anaplasma spp. in dogs, and few available acaricides on the market currently repel ticks (58). Most acaricides rely on transfer of the drug during the tick bite to kill the tick within hours. This is protective against $B$. burgdorferi which requires a prolonged attachment time (59), but may not protect against pathogens, such as Anaplasma spp., that may transmit in a shorter time period (60). As a result, routine practices of preventative care in historically endemic regions may not fully protect against Anaplasma spp., and as a result, yield an increase in seroprevalence.

The evidence of increasing exposure to the tick-borne pathogens from this analysis is notable for several reasons. First, increasing seroprevalence suggests that utilization and compliance with recommended year-round use of preventative measures continues to be inadequate in some areas (57), particularly in established endemic regions and neighboring areas. These observations reinforce the concept that veterinarians and pet owners within these regions should recognize the persistent and growing risk of exposure, and implement appropriate preventative measures. Second, even in the presence of acaricides, prompt removal of ticks is strongly recommended to prevent pathogen transmission. Third, given the dynamic nature of tick-borne diseases, veterinarians practicing in regions proximate to endemic areas should adjust screening and preventative care protocols accordingly. Similarly, emphasis should be placed on vaccinating dogs at risk for Lyme disease prior to exposure (61), and the aforementioned areas of increasing seroprevalence provide veterinary practitioners with evidence-based recommendations for use of Lyme disease vaccines against emerging disease. Even in areas where the trends were not increasing, it is imperative that veterinarians and pet owners recognize that dogs are still at risk of exposure, particularly in endemic regions, and that preventative measures and testing should not be discontinued.

Supporting the evidence that dogs can act as sentinels in human vector-borne disease $(15,16)$, is the similarity in temporal trends between this study and incidence rates of human cases reported by the Centers for Disease Control and Prevention (CDC) and other researchers. Specifically, in regions with strong positive trends in canine Anaplasma spp. seroprevalence (i.e., Pennsylvania and northward, Figure 5), positive trends were observed during the same time period in the reported incidencerate of human anaplasmosis $(62,63)$. Similar trends have been noted for human B. burgdorferi cases in Virginia and West Virginia $(8,64)$.

Although the focus of this study was identification of locations where the seroprevalence of B. burgdorferi and Anaplasma spp. was increasing, we noted several areas of that had decreases in seroprevalences. There was a remarkable cluster of counties that had a decrease in B. burgdorferi within the northeastern US, predominately along the Atlantic coast. This occurred for Anaplasma spp. seroprevalence to a lesser extent. The upper Midwest experienced very little increase, which was surprising given the historical endemicity. Instead, most counties experienced a stable or decrease in seroprevalence for both pathogens. However, similar results were obtained by Dewage et al. who analyzed canine serologic data collected during an earlier time period (2010-2017) (54). Finally, these changes are supported by trends that are observed in CDC-reported human cases of Lyme disease and anaplasmosis, lending further support to the use of dogs as sentinels for these, and possibly other, vectorborne pathogens $(15,16)$. Recently, several states within the Northeast have reported decreases in the number of human cases reported annually (65). In Wisconsin and Minnesota, trends of human incidence appeared to be stable during this study period (66). It is important to note that these are short term trends, and the long-term implications of these trends are unknown at this time. It is possible that public education and use of preventative practices in these endemic areas may be reducing the risk of exposure and thus reducing the incidence of infection (67). However, knowledge and use of these practices vary within and outside endemic areas $(68,69)$.

This study focused on two important pathogens associated with Ixodes scapularis, B. burgdorferi, and Anaplasma spp., which have large amounts of exposure data available through veterinary testing; however, this tick species is also a known vector for several human and zoonotic pathogens including Babesia microti, Borrelia miyamotoi, Ehrlichia muris subsp. eauclarensis, Powassan encephalitis virus (70), and the recently discovered $B$. mayonii (71). As we point out above, the seroprevalence of $B$. burgdorferi is, in part, a sentinel for the changing population of Ixodes spp. and, as a result, should compel both human and veterinary medical practitioners to be cognizant of the potential changes in incidence and spatial distribution of all pathogens carried by Ixodes spp. $(15,16)$. The known ranges for many of these pathogens, e.g., E. muris eauclarensis (72) and B. mayonii (73), are currently restricted to a small region, so future 
research is needed to determine what ecological factors drive the presence and distribution of these pathogens and whether there are any correlations with other pathogens transmitted by Ixodes spp.

Finally, there are limitations to this analysis. The temporal trends presented do not explicitly show spatial spread of B. burgdorferi or Anaplasma spp. seroprevalence over the study time period and the results here should not be interpreted as spatial change. The data are from a population of dogs under the care of a veterinarian, and so it is reasonable to assume that these dogs are more likely to be well-cared for and more likely to be provided preventative and medical care. As a result, these results may not reflect changes in higher risk populations of dogs (e.g., shelter and rescue dogs). The trends presented here reflect not only the change in the distribution and prevalence of the pathogens, but also changes in testing and preventative practices, both of which should be considered when interpreting these results. Finally, our data do not include the Canadian territories and provinces. However, there is no physical barrier between the northern US and southern Canadian border to prevent the movement of ticks and B. burgdorferi reservoir hosts. There is a growing recognition of canine Lyme disease in Canada along with increased geographic distribution and density of Ixodes scapularis, increased numbers of B. burgdorferiinfected ticks, increased human cases (74) and increased seroprevalence in dogs $(75,76)$. Canadian veterinarians and human healthcare providers should take the same precautions as those in the USA practicing in these transitional zones.

\section{CONCLUSION}

The results of this analysis support the increase in seroprevalence within currently recognized high incidence regions of $B$. burgdorferi and Anaplasma spp. in the United States and in the regions immediately surrounding those high incidence areas. Veterinarians and pet owners should take the appropriate precautions to prevent exposure to dogs. Although this analysis does not identify specific risk factors associated with the increasing seroprevalence, there are preventative measures veterinarians and pet owners can take to reduce the risk of exposure and infection. Year-round tick preventative is recommended, particularly in areas where ticks are active into the fall and early spring. Similarities between the trends in exposure to vector-borne pathogens in both the

\section{REFERENCES}

1. The Companion Animal Parasite Council. Parasite Prevalence Maps. Available online at: https://www.capcvet.org/maps (2019).

2. Qurollo BA, Chandrashekar R, Hegarty BC, Beall MJ, Stillman BA, Liu J, et al. A serological survey of tick-borne pathogens in dogs in North America and the Caribbean as assessed by Anaplasma phagocytophilum, A. platys, Ehrlichia canis, E. chaffeensis, E. ewingii, and Borrelia burgdorferi species-specific peptides. Infect Ecol Epidemiol. (2014) 4:246-99. doi: 10.3402/iee.v4.24699 canine and human populations support the use of canine data when estimating the risk of exposure to humans and should be considered when developing predictive models for either population.

\section{DATA AVAILABILITY STATEMENT}

The data presented in this study can be found online. The Borrelia data can be found at https://capcvet.org/maps/\#2020/all/ lyme-disease/dog/united-states/ while the Anaplasma data can be found at https:/capcvet.org/maps/\#2020/all/anaplasmosis/ dog/united-states/.

\section{AUTHOR CONTRIBUTIONS}

SS, CM, and DB conceptualized and developed the model. SS wrote the methods. JG, SN, and MY contributed to the interpretation the results and writing of the manuscript. JG, SS, $\mathrm{CM}, \mathrm{DB}, \mathrm{SN}$, and MY reviewed and edited the manuscript. All authors read and approved the final manuscript.

\section{FUNDING}

All authors would like to thank and acknowledge their funding. DB was funded by the National Science Foundation (NSF) grants CMMI-1934438, EEC-1744497, and OIA-1826715. CM was supported by NSF grant OIA-1826715, National Institutes of Health grant Al121351, and Department of Defense's Office of Naval Research grant N00014-19-1-2295. JG and SS were funded by the Companion Animal Parasite Council (CAPC). The funders had no role in any part of the study or manuscript.

\section{ACKNOWLEDGMENTS}

With much gratitude, we thank IDEXX Laboratories Inc. for sharing these data and allowing studies such as this and many more.

\section{SUPPLEMENTARY MATERIAL}

The Supplementary Material for this article can be found online at: https:/www.frontiersin.org/articles/10.3389/fvets. 2020.561592/full\#supplementary-material 
6. Kugeler KJ, Farley GM, Forrester JD, Mead PS. Geographic distribution and expansion of human Lyme disease, United States. Emerg Infect Dis. (2015) 21:1455-7. doi: 10.3201/eid2108.141878

7. Bacon RM, Kugeler KJ, Mead PS, Centers for Disease Control and Prevention. Surveillance for Lyme disease-United States, 1992-2006. MMWR Surveill Summ. (2008) 57:1-9. Available online at: https://www.cdc.gov/mmwr/ preview/mmwrhtml/ss5710al.htm

8. Lantos PM, Nigrovic LE, Auwaerter PG, Fowler VG, Ruffin F, Brinkerhoff RJ, et al. Geographic expansion of Lyme disease in the southeastern United States, 2000-2014. Open Forum Infect Dis. (2015) 2:ofv143. doi: 10.1093/ofid/ofv143

9. Lantos PM, Tsao J, Nigrovic LE, Auwaerter PG, Fowler VG, Ruffin F, et al. Geographic expansion of Lyme disease in Michigan, 2000-2014. Open Forum Infect Dis. (2017) 4:ofw269. doi: 10.1093/ofid/ofw269

10. Barbour AG, Fish D. The biological and social phenomenon of Lyme disease. Science. (1993) 260:1610-6. doi: 10.1126/science.8503006

11. Eisen RJ, Eisen L, Beard CB. County-scale distribution of Ixodes scapularis and Ixodes pacificus (Acari: Ixodidae) in the Continental United States. J Med Entomol. (2016) 53:349-86. doi: 10.1093/jme/tjv237

12. Kilpatrick AM, Dobson AD, Levi T, Salkeld DJ, Swei A, Ginsberg HS, et al. Lyme disease ecology in a changing world: consensus, uncertainty and critical gaps for improving control. Philos Trans R Soc Lond Ser B Biol Sci. (2017) 372:2016-117. doi: 10.1098/rstb.2016.0117

13. Levi T, Keesing F, Holt RD, Barfield M, Ostfeld RS. Quantifying dilution and amplification in a community of hosts for tick-borne pathogens. Ecol Appl. (2016) 26:484-98. doi: 10.1890/15-0122

14. Demma LJ, Traeger M, Blau D, Gordon R, Johnson B, Dickson J, et al. Serologic evidence for exposure to Rickettsia rickettsii in eastern Arizona and recent emergence of Rocky Mountain spotted fever in this region. VectorBorne Zoonot Dis. (2006) 6:423-9. doi: 10.1089/vbz.2006.6.423

15. Mead P, Goel R, Kugeler K. Canine serology as adjunct to human Lyme disease surveillance. Emerg Infect Dis. (2011) 17:1710. doi: 10.3201/1709.110210

16. Liu Y, Nordone SK, Yabsley MJ, Lund RB, McMahan CS, Gettings JR. Quantifying the relationship between human Lyme disease and Borrelia burgdorferi exposure in domestic dogs. Geospat Health. (2019) 14:111-20. doi: 10.4081 /gh. 2019.750

17. Self SCW, McMahan CS, Brown DA, Lund RB, Gettings JR, Yabsley MJ. A large-scale spatio-temporal binomial regression model for estimating seroprevalence trends. Environmetrics. (2018) 2018:e2538. doi: $10.1002 /$ env. 2538

18. Littman MP, Gerber B, Goldstein RE, Labato MA, Lappin MR, Moore GE. ACVIM consensus update on Lyme borreliosis in dogs and cats. J Vet Intern Med. (2018) 32:887-903. doi: 10.1111/jvim.15085

19. Ijdo JW, Sun W, Zhang Y, Magnarelli LA, Fikrig E. Cloning of the gene encoding the 44-kilodalton antigen of the agent of human granulocytic ehrlichiosis and characterization of the humoral response. Infect Immun. (1998) 66:3264-9. doi: 10.1128/IAI.66.7.3264-3269.1998

20. Stillman BA, Monn M, Liu J, Thatcher B, Foster P, Andrews B, et al. Performance of a commercially available in-clinic ELISA for detection of antibodies against Anaplasma phagocytophilum, Anaplasma platys, Borrelia burgdorferi, Ehrlichia canis, and Ehrlichia ewingii and Dirofilaria immitis antigen in dogs. J Am Vet Med Assoc. (2014) 245:80-6. doi: $10.2460 /$ javma.245.1.80

21. Carpenter RD, Maloney K. (12) Patent Application Publication (10) Pub. No.: US 2006/0222585 A1 Figure 1. (2015). Available online at: https://patentimages.storage.googleapis.com/44/54/86/2b16e39b62a234/ US20150323531A1.pdf

22. Liang FT, Aberer E, Cinco M, Gern L, Hu CM, Lobet YN, et al. Antigenic conservation of an immunodominant invariable region of the VlsE lipoprotein among European pathogenic genospecies of Borrelia burgdorferi SL. J Infect Dis. (2000) 182:1455-62. doi: 10.1086/315862

23. Wagner B, Freer H, Rollins A, Garcia-Tapia D, Erb HN, Earnhart C, et al. Antibodies to Borrelia burgdorferi OspA, OspC, OspF and C6 antigens as markers for early and late infection in dogs. Clin Vaccine Immunol. (2012) 19:CVI-05653. doi: 10.1128/CVI.05653-11

24. Banerjee S, Gelfand AE, Finley AO, Sang H. Gaussian predictive process models for large spatial datasets. J R Stat Soc Ser B. (2008) 70:825-48. doi: $10.1111 / j .1467-9868.2008 .00663 . x$
25. Martínez-Beneito MA, López-Quilez A, Botella-Rocamora P. An autoregressive approach to spatio-temporal disease mapping. Stat Med. (2008) 27:2874-89. doi: 10.1002/sim.3103

26. Rushworth A, Lee D, Mitchell R. A Spatio-temporal model for estimating the long-term effects of air pollution on respiratory hospital admissions in Greater London. Spat Spatio-Tempor Epidemiol. (2014) 10:29-38. doi: 10.1016/j.sste.2014.05.001

27. Besag J. Spatial interaction and the statistical analysis of lattice systems [Journal Article]. J $R$ Stat Soc Ser B. (1974) 36:192-236. doi: 10.1111/j.2517-6161.1974.tb00999.x

28. Lawson A. Bayesian Disease Mapping: Hierarchical Modeling in Spatial Epidemiology. Boca Raton, FL: CRC Press (2018). doi: 10.1201/9781351271769

29. Martínez-Beneito M, Botella-Rocomora P. Disease Mapping: From Foundations to Multidimensional Modeling. Boca Raton, FL: Apple Academic Press, Inc. (2019). doi: 10.1201/9781315118741

30. Bradley AP. The use of the area under the ROC curve in the evaluation of machine learning algorithms. Pattern Recogn. (1997) 30:1145-59. doi: 10.1016/S0031-3203(96)00142-2

31. Fawcett T. An introduction to ROC analysis. Pattern Recogn Lett. (2006) 27:861-74. doi: 10.1016/j.patrec.2005.10.010

32. Mandrekar JN. Receiver operating characteristic curve in diagnostic test assessment. J Thorac Oncol. (2010) 5:1315-6. doi: 10.1097/JTO.0b013e3181ec173d

33. Makowski D, Ben-Shachar MS, Chen SHA, Lüdecke D. Indices of effect existence and significance in the Bayesian framework. Front Psychol. (2019) 10:2767. doi: $10.3389 /$ fpsyg.2019.02767

34. Sjölander A, Vansteelandt S. Frequentist versus Bayesian approaches to multiple testing. Eur J Epidemiol. (2019) 34:809-21. doi: 10.1007/s10654-019-00517-2

35. Scott JG, Berger JO. Bayes and empirical-Bayes multiplicity adjustment in the variable-selection problem. Ann Stat. (2010) 38:2587-619. doi: 10.1214/10-AOS792

36. Sonenshine D. Range expansion of tick disease vectors in North America: implications for spread of tick-borne disease. Int J Environ Res Public Health. (2018) 15:478. doi: 10.3390/ijerph15030478

37. Gasmi S, Bouchard C, Ogden NH, Adam-Poupart A, Pelcat Y, Rees EE, et al. Evidence for increasing densities and geographic ranges of tick species of public health significance other than Ixodes scapularis in Québec, Canada. PLoS ONE. (2018) 13:e0201924. doi: 10.1371/journal.pone.0201924

38. Eisen RJ, Kugeler KJ, Eisen L, Beard CB, Paddock CD. Tick-borne zoonoses in the United States: persistent and emerging threats to human health. ILAR J. (2017) 58:319-35. doi: 10.1093/ilar/ilx005

39. Lee X, Coyle DR, Johnson DKH, Murphy MW, McGeehin MA, Murphy RJ, et al. Prevalence of Borrelia burgdorferi and Anaplasma phagocytophilum in Ixodes scapularis (Acari: Ixodidae) nymphs collected in managed red pine forests in Wisconsin. J Med Entomol. (2014) 51:694-701. doi: 10.1603/ME13140

40. Aliota MT, Dupuis AP, Wilczek MP, Peters RJ, Ostfeld RS, Kramer LD. The prevalence of zoonotic tick-borne pathogens in Ixodes scapularis collected in the Hudson Valley, New York State. Vector-Borne Zoon Dis. (2014) 14:245-50. doi: 10.1089/vbz.2013.1475

41. Hutchinson ML, Strohecker MD, Simmons TW, Kyle AD, Helwig MW. Prevalence rates of Borrelia burgdorferi (Spirochaetales: Spirochaetaceae), Anaplasma phagocytophilum (Rickettsiales: Anaplasmataceae), and Babesia microti (Piroplasmida: Babesiidae) in host-seeking Ixodes scapularis (Acari: Ixodidae) from Pennsylvania. J Med Entomol. (2015) 52:693-8. doi: 10.1093/jme/tjv037

42. Larson SR, Lee X, Paskewitz SM. Prevalence of tick-borne pathogens in two species of Peromyscus mice common in Northern Wisconsin. J Med Entomol. (2018) 55:1002-10. doi: 10.1093/jme/tjy027

43. Johnson RC, Kodner C, Jarnefeld J, Eck DK, Xu Y. Agents of human anaplasmosis and Lyme disease at Camp Ripley, Minnesota. Vector-Borne Zoon Dis. (2011) 11:1529-34. doi: 10.1089/vbz.2011.0633

44. Liu Y, Watson SC, Gettings JR, Lund RB, Nordone SK, Yabsley MJ, et al. A Bayesian spatio-temporal model for forecasting Anaplasma species seroprevalence in domestic dogs within the contiguous United States. PLoS ONE. (2017) 12:e0182028. doi: 10.1371/journal.pone.0182028 
45. Herrin BH, Zajac AM, Little SE. Confirmation of Borrelia burgdorferi sensu stricto and Anaplasma phagocytophilum in Ixodes scapularis, Southwestern Virginia. Vector-Borne Zoon Dis. (2014) 14:821-4. doi: 10.1089/vbz.2014.1661

46. Stephenson N, Foley J. Parallelisms and contrasts in the diverse ecologies of the Anaplasma phagocytophilum and Borrelia burgdorferi complexes of bacteria in the far western United States. Vet Sci. (2016) 3:26. doi: $10.3390 /$ vetsci3040026

47. Goldstein RE, Eberts, Mj B, Thatcher B, Chandrashekar R, Alleman AR. Performance comparison of SNAP (B) 4Dx (R)Plus and AccuPlex ( 4 for the detection of antibodies to Borrelia burgdorferi and Anaplasma phagocytophilum. Vol. 12 (2014). p. 141-7.

48. Liu J, Drexel J, Andrews B, Eberts M, Breitschwerdt E, Chandrashekar R. Comparative evaluation of 2 in-clinic assays for vector-borne disease testing in dogs. Top Compan Anim Med. (2018) 33:114-8. doi: 10.1053/j.tcam.2018.09.003

49. Chandrashekar R, Beall MJ, Thatcher B, Saucier JM, Tyrrell P, Lappin MR. Serologic responses to peptides of Anaplasma phagocytophilum and Borrelia burgdorferi in dogs infested with wild-caught Ixodes scapularis. Vet J. (2017) 226:6-11. doi: 10.1016/j.tvjl.2017.06.005

50. Little SE. Ehrlichiosis and anaplasmosis in dogs and cats. Vet Clin. (2010) 40:1121-40. doi: 10.1016/j.cvsm.2010. 07.004

51. Gray J, Dantas-Torres F, Estrada-Pe na A, Levin M. Systematics and ecology of the brown dog tick, Rhipicephalus sanguineus. Ticks Tick-Borne Dis. (2013) 4:171-80. doi: 10.1016/j.ttbdis.2012.12.003

52. Pybus MJ, Monismith SW, Kocan AA. Parasitic Diseases of Wild Mammals. Wiley Online Library (2001).

53. Salkeld DJ, Lane RS. Community ecology and disease risk: lizards, squirrels, and the Lyme disease spirochete in California, USA. Ecology. (2010) 91:293-8. doi: 10.1890/08-2106.1

54. Dewage BG, Little S, Payton M, Beall M, Braff J, Szlosek D, et al. Trends in canine seroprevalence to Borrelia burgdorferi and Anaplasma spp. in the eastern USA, 2010-2017. Parasites Vectors. (2019) 12:1-3. doi: 10.1186/s13071-019-3735-x

55. Bisanzio D, Fernández MP, Martello E, Reithinger R, Diuk-Wasser MA. Current and future spatiotemporal patterns of Lyme disease reporting in the Northeastern United States. JAMA Netw Open. (2020) 3:e200319. doi: 10.1001/jamanetworkopen.2020.0319

56. Oliver JD, Bennett SW, Beati L, Bartholomay LC. Range expansion and increasing Borrelia burgdorferi infection of the tick Ixodes scapularis (Acari: Ixodidae) in Iowa, 1990-2013. J Med Entomol. (2017) 54:1727-34. doi: 10.1093/jme/tjx121

57. Lavan RP, Tunceli K, Zhang D, Normile D, Armstrong R. Assessment of dog owner adherence to veterinarians' flea and tick prevention recommendations in the United States using a cross-sectional survey. Parasites Vectors. (2017) 10:284. doi: 10.1186/s13071-017-2217-2

58. Animalytix, editor. Compendium of Veterinary Products - US Edition. North American Compendiums (2018). Available online at: https://bayerall. cvpservice.com/

59. Piesman J, Mather TN, Sinsky R, Spielman A. Duration of tick attachment and Borrelia burgdorferi transmission. J Clin Microbiol. (1987) 25:557-8. doi: 10.1128/JCM.25.3.557-558.1987

60. des Vignes F, Piesman J, Heffernan R, Schulze TL, Stafford III KC, Fish D. Effect of tick removal on transmission of Borrelia burgdorferi and Ehrlichia phagocytophila by Ixodes scapularis nymphs. J Infect Dis. (2001) 183:773-8. doi: $10.1086 / 318818$

61. Levy SA. Use of a C6 ELISA test to evaluate the efficacy of a wholecell bacterin for the prevention of naturally transmitted canine Borrelia burgdorferi infection. Vet Therap. (2002) 3:420-4.

62. Elias SP, Bonthius J, Robinson S, Robich RM, Lubelczyk CB, Smith RP Jr. Surge in anaplasmosis cases in Maine, USA, 2013-2017. Emerg Infect Dis. (2020) 26:327. doi: 10.3201/eid2602.190529
63. (NNDSS) NNDSS. Nationally Notifiable Infectious Diseases and Conditions, United States. (2020). Available online at: https://wonder.cdc.gov/nndss/ nndss_annual_tables_menu.asp

64. Gupta R. HEALTH ADVISORY \#141 Dramatic Increase in Lyme Disease Cases. West Virginia Department of Health \& Human Resources (2017).

65. Centers for Disease Control and Prevention. Data and Statistics: Lyme Disease. (2019). Available online at: https://www.cdc.gov/lyme/stats/tables.html

66. Murphy DS, Lee X, Larson SR, Johnson DKH, Loo T, Paskewitz SM. Prevalence and distribution of human and tick infections with the Ehrlichia muris-like agent and Anaplasma phagocytophilum in Wisconsin, 2009-2015. Vector-Borne Zoon Dis. (2017) 17:229-36. doi: 10.1089/vbz.2016.2055

67. Connally NP, Durante AJ, Yousey-Hindes KM, Meek JI, Nelson RS, Heimer R. Peridomestic Lyme disease prevention: results of a population-based case-control study. Am J Prevent Med. (2009) 37:201-6. doi: 10.1016/j.amepre.2009.04.026

68. Butler AD, Sedghi T, Petrini JR, Ahmadi R. Tick-borne disease preventive practices and perceptions in an endemic area. Ticks Tick-Borne Dis. (2016) 7:331-7. doi: 10.1016/j.ttbdis.2015.12.003

69. Aenishaenslin C, Bouchard C, Koffi JK, Ogden NH. Exposure and preventive behaviours toward ticks and Lyme disease in Canada: results from a first national survey. Ticks Tick-Borne Dis. (2017) 8:112-8. doi: 10.1016/j.ttbdis.2016.10.006

70. Nelder MP, Russell CB, Sheehan NJ, Sander B, Moore S, Li Y, et al. Human pathogens associated with the blacklegged tick Ixodes scapularis: a systematic review. Parasites Vectors. (2016) 9:265. doi: 10.1186/s13071-016-1529-y

71. Dolan MC, Hojgaard A, Hoxmeier JC, Replogle AJ, Respicio-Kingry LB, Sexton C, et al. Vector competence of the blacklegged tick, Ixodes scapularis, for the recently recognized Lyme borreliosis spirochete Candidatus Borrelia mayonii. Ticks Tick-Borne Dis. (2016) 7:665-9. doi: 10.1016/j.ttbdis.2016.02.012

72. Stromdahl E, Hamer S, Jenkins S, Sloan L, Williamson P, Foster E, et al. Comparison of phenology and pathogen prevalence, including infection with the Ehrlichia muris-like (EML) agent, of Ixodes scapularis removed from soldiers in the midwestern and the northeastern United States over a 15 year period (1997-2012). Parasites Vectors. (2014) 7:553. doi: 10.1186/PREACCEPT-1577044381141706

73. Pritt BS, Mead PS, Johnson DKH, Neitzel DF, Respicio-Kingry LB, Davis JP, et al. Identification of a novel pathogenic Borrelia species causing Lyme borreliosis with unusually high spirochaetaemia: a descriptive study. Lancet Infect Dis. (2016) 16:556-64. doi: 10.1016/S1473-3099(15)00464-8

74. Bouchard C, Leonard E, Koffi JK, Pelcat Y, Peregrine A, Chilton N, et al. The increasing risk of Lyme disease in Canada. Can Vet J. (2015) 56:693.

75. Herrin BH, Peregrine AS, Goring J, Beall MJ, Little SE. Canine infection with Borrelia burgdorferi, Dirofilaria immitis, Anaplasma spp. and Ehrlichia spp. in Canada, 2013-2014. Parasites Vectors. (2017) 10:244. doi: 10.1186/s13071-017-2184-7

76. Evason M, Stull JW, Pearl DL, Peregrine AS, Jardine C, Buch JS, et al. Prevalence of Borrelia burgdorferi, Anaplasma spp., Ehrlichia spp. and Dirofilaria immitis in Canadian dogs, 2008 to 2015: a repeat cross-sectional study. Parasites Vectors. (2019) 12:64. doi: 10.1186/s13071-019-3299-9

Conflict of Interest: The authors declare that the research was conducted in the absence of any commercial or financial relationships that could be construed as a potential conflict of interest.

Copyright (c) 2020 Gettings, Self, McMahan, Brown, Nordone and Yabsley. This is an open-access article distributed under the terms of the Creative Commons Attribution License (CC BY). The use, distribution or reproduction in other forums is permitted, provided the original author(s) and the copyright owner(s) are credited and that the original publication in this journal is cited, in accordance with accepted academic practice. No use, distribution or reproduction is permitted which does not comply with these terms. 